\title{
Hilbert boundary value problem for generalized analytic functions with a singular line
}

\author{
Pavel Shabalin ${ }^{1[0000-0002-2791-3964]}$, and Rafael Faizov*1[0000-0003-4744-164X] \\ ${ }^{1}$ Kazan State University of Architecture and Engineering, 420043 Kazan, Russia
}

\begin{abstract}
In this paper, we study an inhomogeneous Hilbert boundary value problem with a finite index and a boundary condition on a circle for a generalized Cauchy-Riemann equation with a singular coefficient. To solve this problem, we conducted a complete study of the solvability of the Hilbert boundary value problem of the theory of analytic functions with an infinite index due to a finite number of points of a special type of vorticity. Based on these results, we have derived a formula for the general solution and studied the existence and number of solutions to the boundary value problem of the theory of generalized analytic functions.

Keywords. Generalized analytical functions, Hilbert problem, infinite index, refined zero-order whole functions.
\end{abstract}

\section{Introduction}

Analytical and generalized analytical functions are widely used in modeling various physical and mechanical processes (for example [1, 2]). In particular, the theory of generalized analytic functions has deep applications to mechanical problems of infinitesimal surface bends [3] and problems of stress state of membrane shell theory $[3,4]$. The Riemann boundary value problems (in particular, the jump problem) $[5,6]$ and Hilbert $[7,8]$ play a significant role in this case. We can interpret any infinitesimal bending of the surface as a certain state of stress equilibrium of the shell. This state of the shell can be described by solving a homogeneous equation. We reduce this equation to the Hilbert boundary value problem for analytic functions. The determination of the hydrodynamic pressure on the side surfaces of the shell in the presence of a surface load is also reduced to the Riemann-Hilbert problem of the theory of generalized analytical functions [3], p. 491. So, the boundary value problems for generalized analytical functions are the apparatus for solving mechanical problems, and the methods developed for the boundary value problems of the theory of generalized analytical functions can also be used to solve many nonlinear problems of the general bending problem.

Such well-known mathematicians as A.V. Bitsadze [9], M.I. Višik and O.A. Ladyženskaya [10], V.A. Solonnikov [11], N.A. Zhura and A.P. Soldatov [12, 13], A.P. Soldatov [14], A.B. Rasulov and A.P. Soldatov [15], and others were engaged in boundary value problems for solving systems of differential equations. The theory of boundary value problems for systems of elliptic differential equations turned out to be very promising for applications in mechanics and physics.

\footnotetext{
${ }^{*}$ Corresponding author: rafael.faizov2@gmail.com
} 
In classical theory [3] generalized analytic functions $U(z), z=x+i y$, satisfy in some domain a linear elliptic system of differential equations, which is usually given in the following complex notation:

$$
\partial_{\bar{z}} U-A(z) U+B(z) \bar{U}=F(z), \quad \partial_{\bar{z}}:=\frac{1}{2}\left(\frac{\partial}{\partial x}+i \frac{\partial}{\partial y}\right),
$$

where the coefficients and the right-hand side of the equation are summable functions with some degree $p>2$. Fundamental results in the theory of boundary value problems for solutions of the system of the type (1) are obtained by I.N. Vekua [3, 4]. Notable contributions to the development of this theory were made by J. Nitsche [14], B.V. Boyarsky [15], L.G. MihaîLov [16], A.P. Soldatov [17, 18].

The relaxation of restrictions on the coefficients of equation (1) is an important direction in the development of the theory of generalized analytic functions. Z.D. Usmanov [19] constructed a complete theory of generalized Cauchy-Riemann systems, whose coefficients have a polar singularity of the 1 st order at an isolated inner point of the domain. On the basis of the developed analytical apparatus, he investigated local and global problems of the theory of infinitesimal bends of surfaces with an isolated flattening point. The construction of the solution of the generalized Cauchy-Riemann system with strong singularities of the coefficients at an isolated point is also devoted to the work [20]. The study of solutions of equation (1) in the case when the coefficients of this equation have singular lines is given in the monograph [21], also [22, 23]. We emphasize that the presence of strong singularities in the coefficients of equation (1) is not only a natural development of the classical theory of generalized analytic functions, but is also in demand as models for problems of thin momentless shells, axisymmetric field theory, and deformation problems [9, 3].

Boundary value problems for generalized Cauchy-Riemann equations with singular coefficients were solved by N.R. Radjabov, A.B. Rasulov, A.P. Soldatov, U.S. Fedorov, Bobodzhanova M.A., and others (for example, [24-27]). In these papers, the method for solving the boundary value problem for equation (1) is based on a reduction to a similar problem for analytic functions.In this case (for example, $[28,29]$ ), it is possible to formulate boundary value problems for generalized analytic functions with a singular line, when the coefficients of equation (1) delegate their features to the boundary condition of the problem for analytic functions and turn the latter into a problem with an infinite index. A.B. Rasulov investigated some situations related to this effect, when the boundary value problem of the theory of generalized analytic functions with a finite index has an infinite set of solutions [28, 29].

This article is written in line with these works of A.B. Rasulov. The paper contains a detailed study of the solvability of the Hilbert boundary value problem with finite index for a class of generalized analytic functions with a singular line. The results of the article supplement the research of A.P. Soldatov and A.B. Rasulov [30], who, under certain restrictions on singular coefficients, derived the formula for the general solution of equation (1). This solution was used by the authors in reducing the boundary value problem for equation (1) to a similar problem with a finite index for analytic functions.

By loosening the restrictions on the coefficients from [30], we reduce the solution of the Hilbert problem for generalized analytic functions with a finite index to the problem for analytic functions, but with an infinite index and two points of vorticity with a new type of singularities. We construct a formula for the general solution of the problem, and conduct a complete study of the solvability. 


\section{Materials and methods}

In the unit circle $D=\left\{z=r e^{i \theta}: 0<r<1,0 \leq \theta<2 \pi\right\}, L=\partial D$, the plane of a complex variable $z=x+i y=r e^{i \theta}$ we consider the Hilbert boundary value problem about finding by the boundary condition:

$$
\operatorname{Re}\left[e^{i \alpha(t)} U(t)\right]=f(t), t \in L, \alpha(t), f(t) \in H(L),
$$

solution $U(z)$ of the generalized Cauchy-Riemann system with a singular line:

$$
\partial_{\bar{z}} U-A(z) U=F(z), \quad A(z)=\frac{a(z)}{\bar{z}-z} .
$$

We highlight that equation (3) is a special case of the studied by A.P. Soldatov and A.B. Rasulov in [14] of the generalized Cauchy-Riemann equation:

$$
\begin{gathered}
\partial_{\bar{z}} U-A(z) U+B(z) \bar{U}=F(z), \\
A(z)=\frac{(z-\bar{z}) a(z)}{|z-\bar{z}|^{n+1}}, B(z)=\frac{b(z)}{|z-\bar{z}|^{m}}, n>0,0<m<1,
\end{gathered}
$$

in a singly connected domain $G$ with a smooth border, with singular $(n=1)$ or super-singular $(n>1)$ line. For this equation, a formula for the general solution is derived, and a boundary value problem with a combined boundary condition is set and solved. These conditions combine the features of the linear conjugation problem and the Hilbert problem.

Following [30], we will assume that for $A(z)$ there is such an analytic in $D$ function $\alpha(z)$ for that:

$$
A_{0}(z)=\frac{a(z)-a_{0}(z)}{\bar{z}-z} \in L^{p}(D), p>2 .
$$

In [30] under additional conditions:

$$
a_{0}\left(\tau_{0}\right)=a_{0}\left(\tau_{1}\right)=0, a_{0}(t) \in H(L)
$$

where $\tau_{0}, \tau_{1}$ - points of intersection of the contour $L$ with a singular line (in this article $\tau_{0}=1$, $\tau_{l}=-1$ ), a formula for the general solution of equation (3) is derived, which for the domain $D$ takes the form:

function:

$$
U(z)=e^{\Omega(z)}\left[\left(T\left(e^{-\Omega} F\right)\right)(z)+\phi(z)\right]
$$

$$
\Omega(z)=\left(T A_{0}\right)(z)+\frac{1}{2 \pi i} \int_{L} \frac{a_{0}(t) \ln |t-\bar{t}|}{t-z} d t+a_{0}(z) \ln |z-\bar{z}|,
$$

Vekua integral operator:

$$
\left(T A_{0}\right)(z)=-\frac{1}{\pi} \int_{D} \frac{A_{0}(\zeta) d_{2} \zeta}{\zeta-z}, \quad z \in D
$$

acts [1] from $L^{p}(D), p>2$, to the Helder class $H(\bar{D})$, the function $\phi(z)$ is analytic in the domain $G_{0} \subseteq D$. We emphasize that conditions (5) also guarantee a finite index of the boundary value problem for analytic functions, which is obtained when solving the problem considered in [30] with a combined boundary condition.

In this paper, instead of condition (5), we assume that the following asymptotic formulas are satisfied:

$$
a_{0}(t)=a_{0}\left(\tau_{j} \pm 0\right)+O\left(\left|t-\tau_{j}\right|\right), t \rightarrow \tau_{j} \pm 0, j=0, j=1, a_{0}\left(\tau_{j} \pm 0\right)=\alpha_{j}^{ \pm}+i \beta_{j}^{ \pm} .
$$

Accordingly, we will consider the boundary values of the function $a_{0}(z)$ to be Heldercontinuous on the upper $L^{+}$and lower $L^{-}$arcs of the unit circle, including the ends. This leads to the following changes in the proof of formula (6).

As in [14], we introduce $D_{\varepsilon}{ }^{ \pm}=D \cap\{ \pm \operatorname{Im} z>\varepsilon\}, \varepsilon-$ as a small positive number and denote $D_{\varepsilon}=D_{\varepsilon}{ }^{+} \cup D_{\varepsilon}^{-}$, the boundary of the region $D / D_{\varepsilon}$ is made up of the union $l_{\varepsilon}$ of two segments $l_{\varepsilon}^{ \pm}$and the union $\gamma_{\varepsilon}$ of two arcs of the unit circle $\gamma_{\varepsilon, 0}, \gamma_{\varepsilon, l}$. These circles contain the points $\tau_{0}=1, \tau_{1}=-1$, respectively. We denote by the symbol $T_{\varepsilon} A$ the integral Vekua operator on the 
union of domains $D_{\varepsilon}$. We need to make sure that $\left(T_{\varepsilon} A\right)(z), z \in K$, converges uniformly at $\varepsilon \rightarrow 0$ to the limit of $\Omega(z)$ on any compact $K, K \in D^{+} \cup D^{-}$, where $D^{ \pm}=D \cap\{ \pm \operatorname{Imz}>0\}$. Following [30], we represent $T_{\varepsilon} A$ in the form:

$$
\left(T_{\varepsilon} A\right)(z)=\left(T_{\varepsilon} A_{0}\right)(z)-I_{\varepsilon}(z), \quad I_{\varepsilon}(z):=\frac{1}{\pi} \int_{D_{\varepsilon}} \frac{a_{0}(\zeta)}{\bar{z}-z} \cdot \frac{d_{2} \zeta}{\zeta-z},
$$

and the last integral is understood in the sense of:

$$
I_{\varepsilon}(z)=\lim _{\delta \rightarrow 0} \frac{1}{\pi} \int_{D_{\varepsilon, \delta}} \frac{a_{0}(\zeta)}{\bar{z}-z} \cdot \frac{d_{2} \zeta}{\zeta-z}, \quad D_{\varepsilon, \delta}=D_{\varepsilon} \cap\{|\zeta-z| \geq \delta\} .
$$

We transform the right-hand side of the equality according to Green's formula:

$$
\frac{1}{\pi} \int_{D_{\varepsilon, \delta}} \frac{a_{0}(\zeta)}{\bar{z}-z} \cdot \frac{d_{2} \zeta}{\zeta-z}=-\frac{1}{2 \pi i}\left(\int_{L \backslash \gamma_{\varepsilon}}+\int_{l_{\varepsilon}} \mid \frac{a_{0}(t) \ln |t-\bar{t}|}{t-z} d t+\frac{1}{2 \pi i} \int_{|z-t|=\delta} \frac{a_{0}(t) \ln |t-\bar{t}|}{t-z} d t,\right.
$$

the segment $l_{\varepsilon}^{-}$is oriented negatively. After moving to the limit in $\delta \rightarrow 0$ we get:

$$
I_{\varepsilon}(z)=-\frac{1}{2 \pi i}\left(\int_{L \backslash \gamma_{\varepsilon}}+\int_{l_{\varepsilon}}\right) \frac{a_{0}(t) \ln |t-\bar{t}|}{t-z} d t-a_{0}(z) \ln |z-\bar{z}| .
$$

We will take into account that:

$$
\frac{1}{2 \pi i} \int_{l_{\varepsilon}} \frac{a_{0}(t) \ln |t-\bar{t}|}{t-z} d t=\frac{\ln 2 \varepsilon}{2 \pi i} \int_{l_{\varepsilon}} \frac{a_{0}(t)}{t-z} d t,
$$

now, for $z \in K$ and sufficiently small values of $\varepsilon$ by Cauchy's theorem, we have:

$$
\frac{\ln 2 \varepsilon}{2 \pi i} \int_{l_{\varepsilon}} \frac{a_{0}(t)}{t-z} d t=\frac{\ln 2 \varepsilon}{2 \pi i} \int_{\gamma_{\varepsilon}} \frac{a_{0}(t)}{t-z} d t
$$

For $z \in K$ and for $\varepsilon \rightarrow 0$, using the Helder continuity on the arcs (including ends) $L^{+}$and $L^{-}$of the function $a_{0}(t)$, we derive:

$$
\frac{\ln 2 \varepsilon}{2 \pi i} \int_{\gamma_{\varepsilon}} \frac{a_{0}(t)}{t-z} d t \rightarrow 0
$$

Thus,

$$
\lim _{\varepsilon \rightarrow 0} \frac{1}{\pi} \int_{D_{\varepsilon}} \frac{a_{0}(\zeta)}{\bar{z}-z} \cdot \frac{d_{2} \zeta}{\zeta-z}=-a_{0}(z) \ln |z-\bar{z}|-\frac{1}{2 \pi i} \int_{L} \frac{a_{0}(t) \ln |t-\bar{t}|}{t-z} d t,
$$

in this case, the Cauchy-type integral with two points of discontinuity of the density of the logarithmic type has [31] near the point $\tau_{j}$ of the form:

$$
\begin{aligned}
& \Phi(z):=\frac{1}{2 \pi i} \int_{L} \frac{a_{0}(t) \ln |t-\bar{t}|}{t-z} d t=\left[a_{0}\left(\tau_{j}-0\right)-a_{0}\left(\tau_{j}+0\right)\right] \frac{\ln ^{2}\left(z-\tau_{j}\right)}{4 \pi i}+ \\
& +\left[a_{0}\left(\tau_{j}-0\right)+a_{0}\left(\tau_{j}+0\right)\right] \frac{\ln \left(z-\tau_{j}\right)}{2}+\Psi(z),
\end{aligned}
$$

where $\Psi(z)$ - is an analytic in $D$ function tending to a certain limit at $z \rightarrow \tau_{j}, j=0, j=1$. In accordance with equality (7), (9) the function $\Omega(z)$ has the form of a point $\tau_{j}$ the following asymptotic representation:

$$
\begin{aligned}
& \Omega(z)=\frac{\beta_{j}^{-}-\beta_{j}^{+}}{4 \pi} \ln ^{2}\left|z-\tau_{j}\right|+\left(\frac{\alpha_{j}^{-}-\alpha_{j}^{+}}{2 \pi} \arg \left(z-\tau_{j}\right)+\frac{\alpha_{j}^{-}+\alpha_{j}^{+}}{2}\right) \ln \left|z-\tau_{j}\right| \\
& -i\left[\frac{\alpha_{j}^{-}-\alpha_{j}^{+}}{4 \pi} \ln ^{2}\left|z-\tau_{j}\right|-\left(\frac{\beta_{j}^{-}-\beta_{j}^{+}}{2 \pi} \arg \left(z-\tau_{j}\right)+\frac{\beta_{j}^{-}+\beta_{j}^{+}}{2}\right) \ln \left|z-\tau_{j}\right|\right] \\
& +a_{0}(z) \ln |z-\bar{z}|+O(1) .
\end{aligned}
$$


Here, by $\arg \left(z-\tau_{j}\right)$ we mean a continuous branch in the domain $D$, whose boundary values satisfy the equalities:

$$
\arg \left(e^{i \theta}-\tau_{j}\right)= \begin{cases}\left(3 \pi+\theta+\theta_{j}\right) / 2, & 0 \leq \theta<\theta_{j}, \\ \left(\pi+\theta+\theta_{j}\right) / 2, & \theta_{j}<\theta \leq 2 \pi\end{cases}
$$

Now we repeat the calculations from [14] and derive the formula for the general solution of equation (3) in the form (6).

Using formula (6), we derive a formula for the general solution of the Hilbert boundary value problem (2) for solutions $U(z)$ of differential equation (3) in the class $A$ of functions $U(z)$ with a product $U e^{-C \Omega}$, bounded in $\bar{D}$ with some $C \geq 1$.

\section{Results}

After we substitute function (6) into condition (2), we obtain the boundary condition for the Hilbert problem for the analytic function $\phi(z)$ in the disk:

$$
\begin{gathered}
\operatorname{Re}\left[e^{i \alpha^{*}(t)} \phi(t)\right]=f^{*}(t), t \in L, t \neq \tau_{0}, t \neq \tau_{l}, \alpha^{*}(t)=\alpha(t)+\operatorname{Im} \Omega(t), \\
f^{*}(t)=f(t) e^{-\operatorname{Re} \Omega(t)}-\operatorname{Re}\left[e^{i \alpha^{*}(t)}\left(T\left(e^{-\Omega} F\right)\right)(t)\right] .
\end{gathered}
$$

After we pass in formula (9) to the limit in $z \rightarrow t, t \in L$, near singular points and introduce the notation $\Delta_{0}^{+}=\frac{3}{4} \alpha_{0}^{-}+\frac{9}{4} \alpha_{0}^{+}, \Delta_{0}^{-}=\frac{13}{4} \alpha_{0}^{-}-\frac{1}{4} \alpha_{0}^{+}, \Delta_{1}^{+}=\frac{5}{4} \alpha_{1}^{-}+\frac{7}{4} \alpha_{1}^{+}, \Delta_{1}^{-}=\frac{15}{4} \alpha_{1}^{-}-\frac{3}{4} \alpha_{1}^{+}$, we get:

$$
\begin{gathered}
\operatorname{Re} \Omega(t)=\frac{\beta_{0}^{-}-\beta_{0}^{+}}{4 \pi} \ln ^{2}\left|t-\tau_{0}\right|+\Delta_{0}^{+} \ln \left|t-\tau_{0}\right|+O(1), \quad t \rightarrow \tau_{0}+0, \\
\operatorname{Re} \Omega(t)=\frac{\beta_{0}^{-}-\beta_{0}^{+}}{4 \pi} \ln ^{2}\left|t-\tau_{0}\right|+\Delta_{0}^{-} \ln \left|t-\tau_{0}\right|+O(1), \quad t \rightarrow \tau_{0}-0, \\
\operatorname{Im} \Omega(t)=\frac{\alpha_{0}^{+}-\alpha_{0}^{-}}{4 \pi} \ln ^{2}\left|t-\tau_{0}\right|+O\left(\ln \left|t-\tau_{0}\right|\right)+O(1), \quad t \rightarrow \tau_{0}+0, \\
\operatorname{Im} \Omega(t)=\frac{\alpha_{0}^{+}-\alpha_{0}^{-}}{4 \pi} \ln ^{2}\left|t-\tau_{0}\right|+O\left(\ln \left|t-\tau_{0}\right|\right)+O(1), \quad t \rightarrow \tau_{0}-0 . \\
\operatorname{Re} \Omega(t)=\frac{\beta_{1}^{-}-\beta_{1}^{+}}{4 \pi} \ln ^{2}\left|t-\tau_{1}\right|+\Delta_{1}^{+} \ln \left|t-\tau_{1}\right|+O(1), \quad t \rightarrow \tau_{1}+0, \\
\operatorname{Re} \Omega(t)=\frac{\beta_{1}^{-}-\beta_{1}^{+}}{4 \pi} \ln ^{2}\left|t-\tau_{1}\right|+\Delta_{1}^{-} \ln \left|t-\tau_{1}\right|+O(1), \quad t \rightarrow \tau_{1}-0, \\
\operatorname{Im} \Omega(t)=\frac{\alpha_{1}^{+}-\alpha_{1}^{-}}{4 \pi} \ln ^{2}\left|t-\tau_{1}\right|+O\left(\ln \left|t-\tau_{1}\right|\right)+O(1), \quad t \rightarrow \tau_{1}+0, \\
\operatorname{Im} \Omega(t)=\frac{\alpha_{1}^{+}-\alpha_{1}^{-}}{4 \pi} \ln ^{2}\left|t-\tau_{1}\right|+O\left(\ln \left|t-\tau_{1}\right|\right)+O(1), \quad t \rightarrow \tau_{1}-0 .
\end{gathered}
$$

Thus, we reduced the Hilbert problem with a finite index for solving equation (1) to the Hilbert problem (11) with an infinite index and two logarithmic vorticity points for the analytic function. Since the Hilbert boundary value problem with such a vorticity character has not yet been studied, we will carry out the solution of the problem in detail. The boundary condition of the problem, taking into account the formulas (9), (12)-(15) and the equality $\ln \mid t$ $-\bar{t}=2 \ln \left|t-t_{0}\right|+2 \ln \left|t-t_{1}\right|-2 \ln 2$, we will rewrite in the form:

$$
\operatorname{Re}\left\{e^{\Phi(t)+2 a_{0}(t) \ln \left(\left(t-\tau_{0}\right)\left(t-\tau_{l}\right)\right)} e^{\Gamma^{+}(t)} \phi(t)\right]=f^{*}(t) e^{\Gamma_{0}(t)} e^{\operatorname{Re}\left[\Phi(t)+2 a o(t) \ln \left(\left(t-\tau_{0}\right)\left(t-\tau_{l}\right)\right)\right]},
$$

where $\Gamma^{+}(t)$ - is the limit value on the contour $L$ of the integral:

$$
\Gamma(z)=\frac{1}{\pi} \int_{L} \frac{\varphi(t) d \tau}{\tau-z}
$$


with a density $\varphi(t)=\alpha(t)+\operatorname{Im}\left[\Omega(t)-\Phi(t)-2 a_{0}(t)\left(\ln \left(t-\tau_{0}\right)+\ln \left(t-\tau_{1}\right)\right)\right]$ continuous throughout on $L$ :

$$
\Gamma_{0}(t)=\frac{1}{\pi} \int_{L} \frac{\varphi(t) d \tau}{\tau-t} .
$$

We will first consider a homogeneous problem:

$$
\operatorname{Re}\left[e^{\Phi(t)+2 a_{0}(t)\left(\ln \left(t-\tau_{0}\right)+\ln \left(t-\tau_{1}\right)\right)} e^{\Gamma^{+}(t)} \phi(t)\right]=0, \quad t \in L, t \neq \tau_{0}, t \neq \tau_{1} .
$$

We will introduce an analytic function in $D$ :

$$
F(z):=i e^{\Phi(z)+2 a_{0}(z)\left(\ln \left(z-\tau_{0}\right)+\ln \left(z-\tau_{1}\right)\right)} e^{\Gamma(z)} \phi(z) .
$$

This function on $L$ by virtue of (17) satisfies the condition:

$$
\operatorname{Im} F^{+}(t)=0 \text {. }
$$

We will express from equality (18) the desired function:

$$
\phi(z)=-i e^{-\Phi(z)-2 a_{0}(z)\left(\ln \left(z-\tau_{0}\right)+\ln \left(z-\tau_{1}\right)\right)} e^{-\Gamma(z)} F(z) .
$$

It is clear that if function (20) is a bounded solution to problem (17), then there must be a function $F(z)$, analytic in $D$. This function satisfies the inequality:

$$
|F(z)| \leq C e^{C_{1} \ln ^{2}\left|z-\tau_{j}\right|-C_{2}|\ln | z-\tau_{j} \mid}, \quad z \in D, z \rightarrow \tau_{j}, j=0 \text { или } j=1,
$$

That is, this function is an exact growth of $\mathrm{T}$ in a semi-neighborhood of the point $\mathrm{T}$, and its boundary values must satisfy condition (19) and the inequality:

$$
|F(t)| \leq C e^{R e \Omega(t)}, t \in L, t \neq \tau_{j}, j=0, j=1 \text {. }
$$

The validity of the inverse statement follows from the generalized maximum principle for analytic functions ([32] p. 456, 457, see also [33]). Thus, it is proved.

\subsection{Theorem 1}

In order for the solution $\phi(z)$ of problem (17) to be bounded in the domain $D$, it is necessary and sufficient that the function $F(z)$, which is included in the formula of the general solution (20), satisfies in $D$ the growth constraints (21) and on the boundary conditions (19) and (22).

It is clear that the existence and set of bounded solutions to problem (17) depends on the existence and set of analytic functions in $D$, and they satisfy conditions (19), (21), and (22). In [35], it is proved that a homogeneous Hilbert problem with $n$ points of vorticity is solvable if and only if all $n$ homogeneous problems with a single point of vorticity are solvable, that is, the solvability of the problem is affected only by the parameters of each point of vorticity. In this case, the solution of the problem with $n$ points of vorticity can be represented as the products of the solutions of these problems with a single point of vorticity. Thus, the solution of problem (17) $\phi(z)=\phi_{0}(z) \phi_{l}(z)$ where $\phi_{j}(z)$ is the general solution of a homogeneous problem with a single point of vorticity $\tau_{j}, j=0, j=1$, is determined by the formula:

$$
\phi_{j}(z)=-i e^{-\Phi_{j}(z)-2 a_{0}(z) \ln \left(z-\tau_{j}\right)} e^{-\Gamma_{j}^{+}(z)} F_{j}(z),
$$

where $F_{j}(z)$ is an analytic function in the domain $D$ that satisfies the conditions:

$$
\operatorname{Im} F_{j}^{+}(t)=0,\left|F_{j}(t)\right| \leq C e^{R e \Omega(t)}, t \in L, \quad\left|F_{j}(z)\right| \leq C e^{C_{1} \ln ^{2} 2_{z}-\tau_{j}\left|-C_{2}\right| \ln \left|z-\tau_{j}\right|}, z \rightarrow \tau_{j} .
$$

Besides $F(z)=F_{0}(z) F_{l}(z)$. If we use these remarks, the following theorem will be proved.

\subsection{Theorem 2}

Homogeneous boundary value problem (17)

a) has no nontrivial bounded solutions if:

$\beta_{0}^{-}-\beta_{0}^{+}<0$, or $\beta_{1}^{-}-\beta_{1}^{+}<0$,

$\beta_{0}^{-}-\beta_{0}^{+}=0$ and $\Delta_{0}^{+}>0$, or $\beta_{0}^{-}-\beta_{0}^{+}=0$ and $\Delta_{0}^{-}>0$, 
$\beta_{0}^{-}-\beta_{1}^{+}=0$ and $\Delta_{1}^{+}>0$, or $\beta_{1}^{-}-\beta_{1}^{+}=0$ and $\Delta_{l}^{-}>0$;

b) has a single solution of the form:

$$
\phi(z)=-i e^{-\Phi(z)-a_{0}(z)\left(\ln \left(z-\tau_{0}\right)+\ln \left(z-\tau_{1}\right)\right)} e^{-\Gamma^{+}(z)} A, A=\text { const, } \operatorname{Im} A=0,
$$

if one of the following two conditions is met $\beta_{0}{ }^{-}-\beta_{0}{ }^{+}=0, \Delta_{0}{ }^{+}=0, \Delta_{0}{ }^{-}<0$ or $\beta_{I^{-}}-\beta_{I}{ }^{+}=0$ and $\Delta_{I}^{-}>0$ or $\beta_{0}^{-}-\beta_{0}{ }^{+}=0, \Delta_{0}^{-}=0, \Delta_{0}{ }^{+}<0$, and one of the conditions is $\beta_{1}^{-}-\beta_{1}^{+}=0, \Delta_{l}^{+}$ $=0, \Delta_{l}^{-}<0$ or $\beta_{I}^{-}-\beta_{l}^{+}=0, \Delta_{l}^{-}=0, \Delta_{I}^{+}<0$;

c) has an infinite set of solutions of the form (20), where $F(z)$ is an analytic function in $D$ that satisfies the conditions (19)-(22), if one of the following two conditions is met $\beta_{0^{-}}-\beta_{0^{+}}$ $>0$, or $\beta_{0}^{-}-\beta_{0}{ }^{+}=0, \Delta_{0}{ }^{+}<0, \Delta_{0}^{-}<0$ and one of the following conditions $\beta_{1}^{-}-\beta_{1}^{+}>0$, or $\beta_{1}^{-}$ $-\beta_{I}^{+}=0, \Delta_{I}^{+}<0, \Delta_{l}^{-}<0$.

We will prove point a) of the theorem. We will assume that $\beta_{j}^{-}-\beta_{j}^{+}<0$ and problem (17) are solvable. The solvability of the homogeneous problem (17) is equivalent to [35] the solvability of the homogeneous Hilbert problem with a point of vorticity $\tau_{l}$ and the homogeneous Hilbert problem with a point of vorticity $\tau_{0}$. The general solution of a homogeneous problem with one singular point $\tau_{j}, j=0.1$ is represented by the formula (23), in which the function $F_{j}(z)$ is subject to the conditions (24). Now we will consider the question of the existence of the function $F_{j}(z)$, for which we will transfer it to the upper halfplane $H^{+}$. We will get the function:

$$
f_{1}(\zeta)=F_{1} \circ z_{1}(\zeta), \quad z_{1}(\zeta)=\tau_{1} \frac{i \zeta+1}{i \zeta-1}, \quad \zeta=\xi+i \eta
$$

This function must meet the following three conditions:

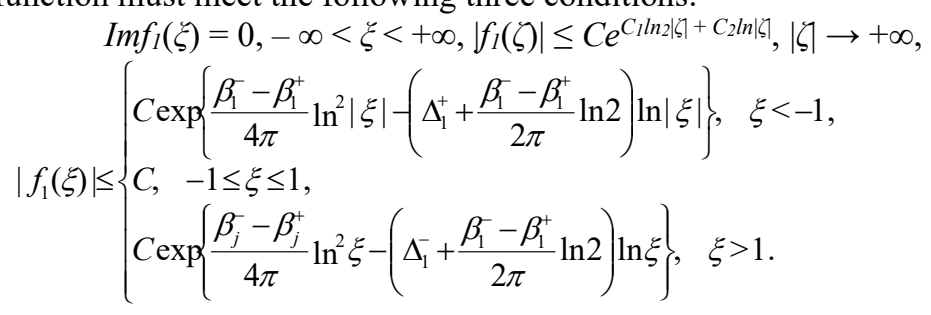

It follows from these conditions that the function $f_{l}(\zeta)$ is a narrowing to the upper halfplane of the whole function:

$$
\widetilde{f}_{1}(\zeta)= \begin{cases}\frac{f_{1}(\zeta),}{f_{1}(\bar{\zeta}),}, \quad \zeta \in H^{+} \\ \hline H^{-}\end{cases}
$$

a refined zero-order $\rho(|\zeta|) \leq \ln \ln ^{2}|\zeta| / \ln |\zeta|$ with a restriction on the growth of its boundary values in the form of inequalities $(25)$. For a function $\widetilde{f}_{1}(\zeta)$ and a plane with a cut along the real semiaxis, we apply the Phragmén - Lindelöf theorem and deduce that under condition $\beta_{j}^{-}-\beta_{j}^{+}<0$ follows $f_{l}(\zeta) \equiv 0, F_{l}(z) \equiv 0$, that is, problem (17) has only a trivial solution. Similarly, we consider the case of the function и $F_{0}(z)$.

Now let $\beta_{j}^{-}-\beta_{j}^{+}=0$. We will consider in detail the case $j=1$. Conditions (21), (22) after passing to the half-plane using the mapping $z_{l}(\zeta)=\tau_{l}(i \zeta+1) /(i \zeta-1)$ now take the form of the inequalities:

$$
\begin{array}{r}
\left|f_{1}(\zeta)\right| \leq C e^{C_{1} \ln |\xi|}, \quad|\zeta| \rightarrow+\infty, \\
\left|f_{1}(\xi)\right| \leq \begin{cases}C \exp \left\{-\Delta_{1}^{+} \ln |\xi|\right\}, & \xi<-1, \\
C, \quad-1 \leq \xi \leq 1, & \\
C \exp \left\{-\Delta_{1}^{-} \ln \xi\right\}, & \xi>1 .\end{cases}
\end{array}
$$

From this, as above, we deduce that if $\Delta_{I}^{+}>0$ or $\Delta_{I^{-}}>0$, then the problem has only a trivial solution. 
We prove point $\mathrm{b}$ ) of Theorem 2 . Let the condition $\beta_{I}^{-}-\beta_{I}^{+}=0, \Delta_{I}^{+}=0, \Delta_{I}^{-}<0$, or condition $\beta_{l}^{-}-\beta_{l}^{+}=0, \Delta_{l}^{-}=0, \Delta_{l}^{+}<0$ be satisfied. We introduce, as above, the function $f_{l}(\zeta), \zeta \in H^{+}$, and the function $\tilde{f}_{1}(\zeta)$. By virtue of the mirror symmetry of the function $\tilde{f}_{1}(\zeta)$ and inequality (26) according to the Phragmén-Lindelöf principle for a plane cut along the real semiaxis with the subsequent application of Liouville's theorem, we obtain $f_{l}(\zeta) \equiv A, A$ = const. But then $F_{I}(z) \equiv A$, that is, we get a unique, up to a constant factor, solution to the homogeneous problem with a vorticity at the point $\tau_{l}$. The uniqueness of the solution of the problem with a vorticity at the point is also proved $\tau_{0}$. The product of these solutions gives a unique solution to problem (17).

We will prove point c) of the theorem 2 . Let the condition $\beta_{j}^{-}-\beta_{j}^{+}>0$ be satisfied. To prove the theorem, we will make sure that formula (20) is meaningful. To do this, we will make sure that there is a function $F(z)$ analytic in the disk $D$ that satisfies conditions (19), (21), and (22). For this we need a whole function:

$$
\hat{f}_{j}(\zeta)=\prod_{k=1}^{\infty}\left(1-\frac{\zeta}{r_{j, k} e^{i \theta_{j}}}\right)\left(1-\frac{\zeta}{r_{j, k} e^{-i \theta_{j}}}\right) .
$$

This function is constructed in [34] on the basis of the example of P.G. Yurov [36]. Here $r_{j, k}=\exp \left\{\left((k-1 / 2) / \lambda_{j}\right)^{1 / \gamma}\right\}, \lambda_{j}>0, \gamma>0, \theta_{j}, 0 \leq \theta_{j} \leq \pi-$ is a fixed quantity. With this choice of parameters for the function $\hat{f}_{j}(\zeta)$ in [20], an asymptotic formula is derived

$$
\ln \left|\hat{f}_{j}(\zeta)\right|=\lambda_{j} \ln ^{\gamma+1}|\zeta|+O\left(\ln ^{\gamma-1}|\zeta|\right), \zeta \rightarrow \infty,
$$

It follows from this formula that for $\gamma=2$ the exact growth of the function at infinity $h_{\tilde{f}_{j}}(|\zeta|)=\ln ^{2}|\zeta|$ and the refined zero order $\rho_{\widetilde{f}_{j}}(|\zeta|)=\ln \ln ^{2}|\zeta| / \ln |\zeta|$. The asymptotic formula for its values on the real axis will take the form:

$$
\ln \left|\hat{f}_{j}(\xi)\right|=\lambda_{j} \ln ^{2}|\xi|+O(1), \quad|\xi| \rightarrow+\infty .
$$

Using the fractional-linear mapping of the unit disc to the upper half-plane:

$$
\zeta_{j}(z)=i \frac{\tau_{j}+z}{\tau_{j}-z}, \quad \tau_{j}=e^{i \theta_{j}},
$$

we construct a regular function in the $\operatorname{disc} \hat{F}_{j}(z)=\hat{f}_{j} \circ \zeta_{j}(z), j=0,1$.

For the function $\hat{F}_{j}(z)$, we can easily obtain an asymptotic representation in a semineighborhood of the point $\tau_{j}$ :

$$
\left|\hat{F}_{j}(z)\right|=\exp \left\{\lambda_{j} \ln ^{2}\left|\tau_{j}-z\right|+\lambda_{j} \ln 4 \ln \frac{1}{\left|\tau_{j}-z\right|}+O(1)\right\}, \quad z \rightarrow \tau_{j}, j=0, j=1 .
$$

From the formula (29) immediately follows the fulfillment of the inequality (21). It follows from formulas (12), (14) that if we choose:

$$
\lambda_{j} \leq \min \left\{\frac{\beta_{j}^{-}-\beta_{j}^{+}}{4 \pi}, \frac{\min \left\{-\Delta_{j}^{-},-\Delta_{j}^{+}\right\}}{\ln 4}\right\},
$$

in formula (27), then the inequality (22) holds for the function $\hat{F}(\zeta)=\hat{F}_{0}(\zeta) \hat{F}_{1}(\zeta)$. It is obvious that the function (27) takes real values on the real axis and, therefore, the functions $\hat{F}_{0}(z), \hat{F}_{1}(z)$ satisfy the condition (19). Then, using the formula (23), we find $\phi_{0}(z), \phi_{1}(z)$ and the desired solution of the homogeneous problem $\phi(z)=\phi_{0}(z) \phi_{l}(z)$.

Finally we consider the case $\beta_{1}^{-}-\beta_{1}^{+}=0, \Delta_{1}^{-}<0, \Delta_{I}^{+}<0$. To prove the theorem in this subsection, it suffices to verify the existence of a solution $\phi_{l}(z)$ to the homogeneous problem with vorticity at the point $\tau_{1}$. As above, this is equivalent to the existence in $H^{+}$of an analytic 
function $f_{l}(\zeta)$. The refined zero order of this function satisfies the inequality $\rho_{f_{1}}(|\zeta|) \leq \ln \ln |\zeta| / \ln |\zeta|$, This function takes real values on the boundary and satisfying condition (26). As such a function, we can take $\tilde{f}_{1}(\zeta)=\zeta^{k_{1}}$ if we choose a positive integer $k_{1}=\min \left\{\left[-\Delta_{1}^{-}\right],\left[-\Delta_{1}^{+}\right]\right\}$. Similarly, but using the conditions $\beta_{0^{-}-} \beta_{0}{ }^{+}=0, \Delta_{0}{ }^{+}<0, \Delta_{0}{ }^{-}<0$, the existence of the solution $\phi_{0}(z)$ is justified.

We now turn to the solution of the inhomogeneous Hilbert problem (11). We will assume that the conditions (C) of Theorem 2 are satisfied, under which the homogeneous boundary value problem (17) is solvable (the situation with condition (b) is elementary).

We will look for the general solution of the inhomogeneous problem in the form of the sum of the general solution of the corresponding homogeneous problem and the particular solution of the inhomogeneous problem. To find the latter, we need some solution of the homogeneous problem (17). For this solution, the function F, which is included in the formula (20) and is defined by the conditions (19), (21) and (22), has the following additional properties:

a) everywhere (except, perhaps, for the points $\tau_{1}, \tau_{2}$ ) on $L$, the condition $F$ is satisfied

$$
\widetilde{F}(t) \neq 0 ;
$$

b) the $\operatorname{limit} \lim _{t \rightarrow \tau_{j}} \frac{e^{\operatorname{Re} \Omega(t)}}{\widetilde{F}(t)}$ exists or goes to infinity of power order $k_{j}<1$;

c) the function $\frac{e^{\operatorname{Re} \Omega(t)}}{\widetilde{F}(t)}$ is Helder continuous on open $\operatorname{arcs} x L^{+}, L^{-}$.

We will search for the function $\widetilde{F}(z)$ in the form $\widetilde{F}(z)=\widetilde{F}_{1}(z) \widetilde{F}_{2}(z)$, where the functions $\widetilde{F}_{1}(z), \widetilde{F}_{2}(z)$ satisfy the conditions (24). We will construct these functions in the following two cases. We will first consider the case of $\beta_{j}^{-}-\beta_{j}^{+}>0, j=0.1$. We introduce a function $\hat{f}_{j}(\zeta)$, which we define in the complex plane $\zeta$ by formula (27). We'll take $\lambda_{\mathrm{j}}=\left(\beta_{j}^{-}\right.$ $\left.-\beta_{j}^{+}\right) / 4 \pi$.

Next, using the mapping (28), we construct a function:

$$
\hat{F}_{j}(z)=\prod_{j=0}^{1} \hat{f}_{j} \circ \zeta_{j}(z)
$$

for the module of which the asymptotic representation (29) is valid. Now we will denote the integer part of the number $x$ with the symbol $\lfloor x\rfloor$. We will consider the following restrictions fulfilled:

$$
\left\lfloor-\Delta_{j}^{+}-\lambda_{j} \ln 4\right\rfloor=\left\lfloor-\Delta_{j}^{-}-\lambda_{j} \ln 4\right\rfloor=r_{j} .
$$

Let $\kappa_{j}^{ \pm}=-\Delta_{j}^{ \pm}-\lambda_{j} \ln 4-r_{j}$. We define the function:

$$
\widetilde{F}_{j}(z)=\hat{F}_{j}(z)\left[i \frac{\tau_{j}+z}{\tau_{j}-z}\right]^{r_{j}}, j=1, j=2
$$

It is easy to check that this function takes real values at the points of the unit circle, and if we compare formulas (29) and (12), (14), it is not difficult to verify the validity of the other two conditions (24). Obviously, also for the constructed function, the fulfillment of additional constraints. Moreover, the function $\frac{e^{R e \Omega(t)}}{\widetilde{F}_{j}(t)}=O\left(\left|t-\tau_{j}\right|^{-\kappa_{j}^{ \pm}}, t \rightarrow \tau_{j} \pm\right.$ is bounded only when the difference $-\Delta_{j}^{ \pm}-\lambda_{j} \ln 4-r_{j}$ is an integer. 
Now we will consider the case when $\beta_{j}^{-}-\beta_{j}^{+}=0$, and the numbers $\Delta_{j}^{+} \leq 0, \Delta_{j}^{-} \leq 0$, and at least one of the inequalities is strict. Here we put $\widetilde{F}_{j}(z)=\left[i \frac{\tau_{j}+z}{\tau_{j}-z}\right]^{r_{j}}, j=1, j=2$, where $r_{j}$ is defined as above.

Finally, we find a function $\widetilde{F}(z)=\widetilde{F}_{1}(z) \widetilde{F}_{2}(z)$ that satisfies the conditions (19), (21), and (22) and the additional constraints a), b), c). По формуле (20) мы построим каноническое решение однородной задачи (17).

$$
\widetilde{\phi}(z)=-i e^{-\Phi(z)-2 a_{0}(z)\left(\ln \left(z-\tau_{0}\right)+\ln \left(z-\tau_{1}\right)\right)} e^{-\Gamma(z)} \widetilde{F}(z) .
$$

Since the function $i e^{i \alpha} \widetilde{\phi}(z)$ takes real values on the boundary circle, we divide the boundary condition (11) of the inhomogeneous problem by $i e^{i \alpha^{*}} \widetilde{\phi}(t)$. Finally, we will get:

$$
R e\left[-i \frac{\phi(t)}{\widetilde{\phi}(t)}\right]=\tilde{f}(t), \quad \widetilde{f}(t)=\frac{f^{*}(t) e^{\operatorname{Re}\left[\Phi(t)+2 a_{0}(t)\left(\ln \left(t-\tau_{0}\right)+\ln \left(t-\tau_{1}\right)\right)\right]} e^{\Gamma_{0}(t)}}{\widetilde{F}(t)} .
$$

We will look for a particular solution to the inhomogeneous problem. This problem has the same sequences of zeros as the function $\widetilde{F}(z)$ and hence $\widetilde{\phi}(z)$.

Therefore, the relation $\phi(z) / \widetilde{\phi}(z)$ for the desired function will be an analytic and bounded function in $D$, with the exception, perhaps, of the points $\tau_{0}, \tau_{l}$, in which a power singularity of order less than one is allowed. Since $\widetilde{f}(t) \in H\left(L^{ \pm}\right)$, and at the points of $\tau_{1}, \tau_{2}$ can have power-law singularities of order less than one, the function $-i \phi(z) / \widetilde{\phi}(z)$ can be represented by the Schwarz formula, therefore:

$$
\phi(z)=-i e^{i \Gamma(z)} \prod_{j=1}^{n} \exp \left\{i g_{j}(z)\right\} \widetilde{F}(z) \frac{1}{\pi} \int_{L} \frac{c_{1}(t) d t}{t-z} .
$$

The last formula gives a particular solution to the inhomogeneous boundary value problem (11), the general solution of which is represented as the sum of the general solution of the corresponding homogeneous problem and the given particular solution. The following is true.

\subsection{Theorem 3}

An inhomogeneous problem (11) is solvable in the class $A$ of analytic functions in $D$ if the corresponding homogeneous problem (17) is solvable. The general solution of the inhomogeneous problem is represented as the sum of the general solution (20) of the homogeneous problem and the particular solution (33) of the inhomogeneous problem.

After we substitute the found solution of the inhomogeneous problem (11) in the formula (6), we get the general solution of the boundary value problem (2).

\section{Discussion}

The article contains a solution and a study of the solvability of the Hilbert boundary value problem for the generalized Cauchy-Riemann equation with a singular coefficient of the form (3). The solution of the boundary value problem is based on the construction of the formula (6) of the general solution of equation (3). This formula allows us to reduce the solution of the boundary value problem (2) with a finite index for a generalized analytic function to the boundary value problem (11) with an infinite index for an analytic function. The main content of our paper is the solution and investigation of the solvability of the boundary value problem (11) with an infinite index and two points of a new type of vorticity. The picture of the 
solvability of problem (11) is contained in Theorems 2, 3. It is described in terms of the characteristics of the singularities of the coefficient and is a picture of the solvability of the boundary value problem (2). If we use the works [20] and [37-39], then we can get a similar result for the Riemann problem.

\section{Conclusions}

We have formulated and solved the inhomogeneous Hilbert boundary value problem for an important special case (3) of the generalized Cauchy-Riemann equation with a singular coefficient. We have obtained a formula for the general solution of this problem. We have found the conditions for the existence and uniqueness of the solution of the boundary value problem. In the case of non-uniqueness of the solution, a complete description of the set of solutions is given. The solution of the Hilbert boundary value problem with an infinite index of the theory of analytic functions is also of independent importance.

\section{References}

1. I.N. Vekua, Bull. Math. Soc. Math. Phys R.P.R 1 2, 233-247 (1957).

2. S.I. Bezrodnykh, Math. Surveys 73 6, 941-1031 (2018).

3. I.N. Vekua, Generalized analytical functions. Nauka, Russian, (1959)

4. I.N. Vekua, Mat Sbornik N S 31 73, 217-314 (1952).

5. W. Haack, II, Math. Nachr, 1-30 (1952).

6. S.N. Timergaliev, Mathematics, 45-61 (2019).

7. S.I. Bezrodnykh, V. Vlasov, Computational Mathematics and Mathematical Physics 60 11, 1898-1914 (2020).

8. S.N. Timergaliev, R.S. Yakushev, Mathematics (2019).

9. A.V. Bitsadze, Some classes of partial differential equations. Nauka, Russian, (1981).

10. M.I. Višik, O.A. Ladyženskaya, Uspehi Mat. Nauki 116 (72), 41-97 (1956).

11. V.A. Solonnikov, Zap. Nauchn. Sem. 444, 133-156 (2016).

12. N.A. Zhura, A.P. Soldatov, Izv. Math 81 3, 542-567 (2017).

13. N.A. Zhura, A.P. Soldatov, Differential Equations 55 6, 815-823 (2019).

14. J. Nitsche, Math. Nachr 7 1, 31-33 (1952).

15. B.V. Boyarskii, Russian Mat. Sb. N.S. 43 85, 451-503 (1957).

16. L.G. Mihailov, Russian Dokl. Akad. Nauk SSSR (N.S.) 112, 13-15 (1957).

17. A.P. Soldatov, Journal of Mathematical Sciences 239 3, 381-411 (2019).

18. A.P. Soldatov, Math. Notes 108 2, 272-276 (2020).

19. Z.D. Usmanov, Generalized Cauchy-Riemann systems with a singular point. Dushanbe, ed. AN, Taj.SSR, (1992).

20. A.B. Rasulov, Math. Notes 108 5, 756-760 (2020).

21. N.R. Radzhabov, Introduction to the theory of partial differential equations with supersingular coefficients. Dushanbe, (1992).

22. A.P. Soldatov, A.B. Rasulov, Differential Equations 54 2, 239-249 (2018).

23. A.B. Rasulov, Differential Equations 53 6, 809-817 (2017).

24. N.R. Radjabov, Siberian Math. J. 13 4, 666 (1972).

25. A.B. Rasulov, M.A. Bobodzhanova, Y.S. Fedorov, Journal of Mathematical Sciences 241 3, 327-339 (2019).

26. A.B. Rasulov, A.P. Soldatov, Complex Variables and Elliptic Equations 64 8, 12751284 (2019).

27. U.S. Fedorov, A.B. Rasulov, Differential Equations 57 1, 140-144 (2021). 
28. I.N. Dorofeeva, A.B. Rasulov, Computational Mathematics and Mathematical Physics 60 10, 1679-1685 (2020).

29. A.B. Rasulov, Differential Equations 40 9, 1364-1366 (2004).

30. A.B. Rasulov, A.P. Soldatov, Differential Equations 52 5, 616-629 (2016).

31. I.M. Melnik, Publishing House of the Mathematical Institute 24, 149-162 (1957).

32. A. Hurwitz, R. Courant, Function theory. Nauka, Russian, (1968).

33. R.B. Salimov, A.Kh. Fatykhov, P.L. Shabalin, Lobachevskii J. Math 38 3, 414-419 (2017).

34. P.L. Shabalin1, A.Kh. Fatykhov, Russian Mathematics 65 1, 57-71 (2021).

35. A.Kh. Fatykhov, P.L. Shabalin, Probl. Anal. Issues Anal 7 25, 30-38 (2018).

36. P.G. Yurov, Izv. Vyssh. Uchebn. Zaved. Mat. 2, 158-163 (1966).

37. E.N. Khasanova, P.L. Shabalin, Russian Math. 63 3, 31-44 (2019).

38. R.B. Salimov, A.Z. Suleimanov, Russian Math. 61 5, 61-65 (2017).

39. R.B. Salimov, Ufa Mathematical Journal 10 1, 80-93 (2018). 